\title{
Curso clínico de la nefropatía membranosa lúpica pura
}

\author{
Rolando Pastén $\mathrm{V}^{\mathbf{1}}$, Loreto Massardo $\mathrm{V}^{\mathbf{1}}$, Helmar Rosenberg \\ $G^{2}$, Francisco Radrigán $A^{3}$, Emilio Roessler $B^{4}$, Andrés \\ Valdivieso $D^{5}$ y Sergio Jacobelli $G^{1}$. \\ Long-Term outcome of type $V$ lupus
membranous glomerulonephritis
}

Background: The long-term outcome of the pure form of WHO type V lupus membranous glomerulonephritis is apparently more benign than that of other forms of lupus glomerulonephritis. However $12 \%$ of such patients progress to terminal renal failure. The presence of proteinuria may be an indication of cytotoxic agents. Aim: To study the clinical long-term outcome of WHO type V lupus membranous glomerulonephritis. Material and methods: A retrospective analysis of all kidney biopsies of a University Pathology Department, with the diagnosis of WHO type $\mathrm{V}$ lupus membranous glomerulonephritis. Review of medical records of patients with the disease and one clinical assessment of all living patients. Results: Between 1973 and 2000, 703 kidney biopsies were done to patients with systemic lupus erythematosus. Of these, 40 were membranous glomerulonephritis and in 33 patients (28 women, age range 6-71 years), data on the evolution and survival was obtained. Nineteen had type Va and the rest type $\mathrm{Vb}$ nephritis. Two presented with renal failure and 11 with proteinuria over $3.5 \mathrm{~g} / 24 \mathrm{~h}$. The median follow-up since the renal biopsy was 63 months (range 1-316). At the end of follow-up, four had a creatinine clearance of less then $15 \mathrm{ml} / \mathrm{h}$ and four a clearance between 15 and $29 \mathrm{ml} / \mathrm{h}$ (one of these received a renal allograft). Eleven (33\%) patients had died, mostly due to infections. Life expectancy at five years with a creatinine clearance over $15 \mathrm{ml} / \mathrm{h}$ was $75 \%$. Bad prognostic factors were an elevated creatinine clearance over $15 \mathrm{ml} / \mathrm{h}$ was $75 \%$. Bad prognostic factors were an elevated creatinine and high blood pressure at the moment of the biopsy. Conclusions: The clinical outcome of these patients was bad. Twelve percent reached a stage of terminal renal failure. This is in contrast with the $3 \%$ progression to a similar stage of proliferative glomerulonephritis treated with i.v. cyclophosphamide. New therapies for this condition must be sought (Rev Méd Chile 2005; 133: 23-32).

(Key-words: Glomerulonephritis, membranous; Kidney failure, chronic; Lupus nephritis)

Recibido el 23 de marzo, 2004. Aceptado en versión corregida el 5 de noviembre, 2004.

${ }^{1}$ Departamento de Inmunología Clínica y Reumatología, ${ }^{2}$ Departamento de Anatomía Patológica. Escuela de Medicina de la Pontificia Universidad Católica de Chile. ${ }^{3}$ Servicio de Medicina. Hospital Dr. Sótero del Río. SSMSO. ${ }^{4}$ Servicio de Nefrología y Medicina Hospital del Salvador. SSMO. Santiago. Chile. ${ }^{5}$ Departamento de Nefrología. Escuela de Medicina de la Pontificia Universidad Católica de Chile.

Este estudio fue financiado en parte por Proyecto Investigación Becarios Residente año 2000. Dirección de Investigación de la Escuela de Medicina de la Pontificia Universidad Católica de Chile.

Correspondencia a: Dra. Loreto Massardo V. Departamento de Inmunología Clínica y Reumatología. Escuela de Medicina de la Pontificia Universidad Católica de Chile. Marcoleta 352. Santiago - Chile. Fax 3624937. Teléfono 6397750. E-mail: massardo@med.puc.cl 
$\mathrm{E}^{1}$ lupus eritematoso generalizado (LEG) es una enfermedad autoinmune y multisistémica, que involucra al riñón en alrededor de la mitad de los enfermos. Este compromiso renal, en algunos casos, conduce a insuficiencia renal terminal (IRT) y es una de las causas principales de muerte. La glomerulonefritis lúpica se cataloga según los hallazgos en la biopsia renal (BR), de acuerdo con la clasificación morfológica revisada de la organización mundial de la salud (OMS), en tipos OMS de I a VI. Esta clasificación correlaciona bien con las manifestaciones clínicas, por lo que se utiliza para sugerir pronóstico e indicar terapia ${ }^{1,2}$.

La glomerulonefritis membranosa lúpica (NML), tipo V de la clasificación OMS, se encuentra con una frecuencia que varía entre $5 \%$ y $25 \%$ del compromiso renal del $\mathrm{LEG}^{1,3,4}$. Se caracteriza por presentar en el microscopio electrónico (ME) un engrosamiento de la membrana basal y depósitos densos subepiteliales e intramembranosos. Se distinguen 2 subtipos tipo $\mathrm{Vb}$ y tipo Va según tengan o no una leve hipercelularidad mesangial, expansión y depósitos mesangiales escasos ${ }^{1,5}$. La patogenia, historia natural y la terapia óptima no son bien conocidas, en parte por el pequeño número de enfermos que abarcan las series y por dificultades propias de la clasificación histológi$\mathrm{ca}^{5,6}$. Clínicamente, se manifiesta con proteinuria en rango nefrótico en alrededor de dos tercios de los casos y con escasos hallazgos serológicos de autoinmunidad $^{7}$. Hay controversia sobre la progresión a IRT, que varía entre 12\% (69 enfermos seguidos por 5 años en revisión que abarcó tres estudios) $)^{5}$ y $42 \%$ (16 enfermos al cabo de 8 años de observación promedio) ${ }^{8}$. La supervivencia renal varía, con tasas entre $93 \%$ y $72 \%$ a 10 años ${ }^{9,10}$. Por esto, algunos autores sugieren que no se requiere un tratamiento inmunosupresor o citotóxico agresivo. Otros, en cambio, recomiendan el uso temprano de citotóxicos en casos con gran proteinuria ${ }^{11,12}$.

En un estudio anterior, realizado en 10 pacientes con nefropatía tipo V, de un total de 124 enfermos con nefritis lúpica, encontramos que la tasa de supervivencia global (renal y vital) a 5 años de los tipos II, III, IV y V fue 83\%, 86\% 64\% y $46 \%$, respectivamente ${ }^{13}$. Por esto, decidimos realizar un estudio con un mayor número de casos seguidos por más tiempo, para lo cual revisamos los casos con tipo $\mathrm{V}$ del registro de biopsias renales de nuestro hospital. El objetivo del presente trabajo fue evaluar retrospectivamente la evolución de 33 enfermos chilenos con lupus eritematoso y nefropatía $\mathrm{Va}$ y $\mathrm{Vb}$, con la participación de 14 centros del país.

\section{PACIENTES Y MÉTODO}

En este estudio retrospectivo, un único patólogo revisó las BR diagnosticadas como glomerulonefritis membranosa lúpica Tipo $\mathrm{Va}$ y $\mathrm{Vb}$ de la $\mathrm{OMS}^{1}$ en el registro del Departamento de Anatomía Patológica del Hospital Clínico de la Universidad Católica. Entre los años 1973 y 2000, hubo 703 BR por LEG, de las que 40 (5,6\%) correspondieron a la forma membranosa lúpica (V OMS). En 33 de los 40 (83\%) casos, pudimos obtener suficientes datos sobre la evolución de los enfermos para análisis, a través del contacto personal o telefónico con los médicos tratantes, quienes autorizaron la revisión de las fichas clínicas y una entrevista con los enfermos. Otros 5 enfermos residían en el extranjero y 2 no se pudieron ubicar. Todos los enfermos cumplieron al menos 4 de los criterios diagnósticos para LEG del American College of Rheumatology durante su evolución ${ }^{14}$. Se aplicó un protocolo escrito, que incluyó datos clínicos y de laboratorio del lupus, de la función renal, del tratamiento recibido y de las causas de muerte. El estudio contó con la aprobación del Comité de Ética de la Facultad de Medicina de la Pontificia Universidad Católica de Chile.

Los datos consignados fueron: hipertensión arterial, definida como una presión arterial por sobre 140/90 mmHg, creatininemia, depuración de creatinina, hematuria ( $>5$ glóbulos rojos por campo de aumento mayor) y proteinuria. Se definió proteinuria en rango nefrótico si ésta era $\geq 3,5 \mathrm{~g} /$ día. No fue posible estudiar la presencia de síndrome nefrótico, porque en su mayoría los datos de proteinemia, albuminemia y colesterol estaban incompletos. La función renal fue agrupada en 4 estadios clínicos, según una adaptación que hemos utilizado ${ }^{13}$ de la clasificación de la función renal para enfermos con lupus, propuesta por Edworthy y $\operatorname{col}^{15}$ (estadio I: creatininemia $<1,3 \mathrm{mg} /$ dl y proteinuria $<1 \mathrm{~g} / \mathrm{l}$; II: creatininemia $<1,3 \mathrm{mg} / \mathrm{dl}$ y proteinuria $\geq 1 \mathrm{~g} / \mathrm{l}$; III: creatininemia entre 1,3 y $3 \mathrm{mg} / \mathrm{dL}$ y cualquier proteinuria; y IV: 
creatininemia $>3 \mathrm{mg} / \mathrm{dL}$ sostenidamente por 1 año o hasta la muerte). La depuración de creatinina fue calculada por la fórmula de Cockcroft ${ }^{16}$ en los casos en que contamos con el peso del paciente. La depuración de la creatinina fue agrupada, a su vez, en 5 estadios clínicos de gravedad según la clasificación de las guías Kidney Disease Outcome Quality Iniatitive (K/DOQI) en Estadio 1: daño renal con una filtración renal glomerular (FRG) normal o aumentada $\geq 90 \mathrm{~mL} / \mathrm{min}$; Estadio 2: daño renal con disminución leve de la FRG entre $60 \mathrm{y}$ $89 \mathrm{~mL} / \mathrm{min}$; Estadio 3: disminución moderada con FRG entre 30 y $59 \mathrm{~mL} / \mathrm{min}$; Estadio 4: disminución grave de la FRG entre 15 y $29 \mathrm{~mL} /$ min y Estadio 5: insuficiencia renal con FRG $<15 \mathrm{~mL} / \mathrm{min} 0$ diálisis $^{17}$. Los datos se registraron en: la primera visita al médico, en el momento de la biopsia renal, en la última visita, a la entrada a hemodiálisis crónica y a la muerte. En los fallecidos, los datos fueron obtenidos de la ficha clínica. Las causas de muerte se agruparon en: lupus activo, complicaciones de la IRT, infecciones y otras. No hubo autopsias.

Se registró el tratamiento prescrito para la nefritis lúpica tipo $\mathrm{V} O \mathrm{OMS}$, sin embargo, este fue variable en dosis y duración entre los médicos tratantes. Para este análisis, el tratamiento fue agrupado en: a) esteroides en dosis mayores 0 iguales a prednisona $50 \mathrm{mg} /$ día o equivalente (Eq.) por al menos un mes b) dosis menores de esteroides, y c) citotóxicos si fueron utilizados por al menos 1 mes.

En noviembre de 2000, los 22 pacientes vivos aceptaron la invitación a participar en el estudio, que consistió en una visita médica y exámenes de laboratorio en 21 casos. Se consignó la actividad de la enfermedad mediante el índice de actividad MEX-SLEDAI (puntaje: $0-32)^{18}$. Se midieron anticuerpos antinucleares (AAN) en células HEp-2, anticuerpos anti-dsDNA por inmunofluorescencia indirecta en crithidia luciliae, complementos C3 y C4, depuración de creatinina, proteinuria de $24 \mathrm{~h}$, sedimento urinario, recuento de leucocitos, recuento de plaquetas y hematocrito.

Histología renal: todas las muestras examinadas en la BR tenían más de 5 glomérulos para observación. Se les hizo microscopia de luz, inmunofluorescencia y ME. Se confirmó en todas el tipo histológico V spuro» de la OMS, 19 eran tipo OMS Va y 14 tipo OMS Vb. En este tipo de nefropatía no se utiliza el índice de actividad y cronicidad del $\mathrm{NIH}^{19}$. En 4 enfermos se practicó una segunda biopsia renal.

Estadística. Los datos se muestran en mediana (intervalo) o en porcentaje. Para el análisis descriptivo de los datos se usó el programa EPI INFO versión 6.0. Para el análisis de expectativa de vida utilizamos el modelo de supervivencia de KaplanMeier. El punto de partida del análisis fue el día de la BR. El punto de término fue la fecha de la muerte $o$ la fecha de entrada a IRT (depuración de creatinina $<15 \mathrm{~mL} / \mathrm{min}$ ) o diálisis crónica si ésta ocurría primero, o el último contacto vivo sin IRT. Los 2 pacientes en hemodiálisis en el momento de la BR fueron excluidos de este análisis. La mediana del seguimiento de la serie fue de 63 meses (1316) con 15 enfermos disponibles para observación a 5 años.

El análisis univariado de los factores de riesgo (sexo; hipertensión arterial; MEX-SLEDAI >10; estadios clínicos de función renal I, II y III; proteinuria $>3 \mathrm{~g} /$ día; hematuria; tratamiento con esteroides; tratamiento citotóxico y Tipos OMS Va $0 \mathrm{Vb}$ ) se hizo mediante tablas de supervivencia de Kaplan-Meier, comparado con la prueba de longrango. Los factores de riesgo continuos (edad, duración de los síntomas, creatininemia y depuración de creatinina) se analizaron con el modelo de Cox en sistema S-PLUS. El nivel de significación elegido fue $\mathrm{p} \leq 0,05$.

\section{RESULTADOS}

Analizamos retrospectivamente el curso clínico de 33 pacientes con nefritis membranosa lúpica. Veintiocho eran mujeres y 5 hombres; la mediana de la edad al momento de la biopsia renal fue 32 años (intervalo 6-71), (Tabla 1).

El tratamiento prescrito tras conocer el resultado de la BR fue prednisona en dosis mayores a 50 mg o Eq, por al menos un mes, en 27 enfermos, en 14 esto se asoció con citotóxicos. Los citotóxicos fueron utilizados por 19 pacientes: azatioprina 9 , ciclofosfamida intravenosa (IV) 6 , ciclofosfamida oral 2, ciclosporina A 1 y leflunomida 1; por tiempos que variaron entre un mes y 5 años. Cinco enfermos combinaron citotóxicos y dosis menores al equivalente de $50 \mathrm{mg}$ de prednisona. 
Tabla 1. Características de 33 pacientes con Lupus Eritematoso $\mathrm{G}$ eneralizado y nefropatía Tipos 0 M S Va y Vb al momento de la biopsia renal

\begin{tabular}{|ll|}
\hline & $\mathrm{N}=33$ \\
\hline Mujeres: hombres & $28: 5$ \\
Edad en años & $32(6-71)$ \\
Meses del inicio del primer síntoma de lupus a la BR & $20(3-233)$ \\
Creatininemia mg/dL & $0,85(0,5-7,9)$ \\
Depuración de creatinina mL/min & $98(9-167)$ \\
Depuración de creatinina (Cockcroft) mL/min* & $95(29-153)$ \\
Proteinuria; gramos en 24 horas & $3,1(0,23-10)$ \\
Hipertensión arterial & $30 \%$ \\
Proteinuria >3, 5 g/24h & $33 \%$ \\
Hematuria (>5 glóbulos rojos por campo de aumento mayor) & $81 \%$ \\
Cilindruria o hematuria & $93 \%$ \\
Anticuerpos antinucleares positivos & $96 \%$ \\
Anticuerpos anti-dsDNA positivos & $47 \%$ \\
Complemento C3 bajo & $43 \%$ \\
Complemento C4 bajo & $50 \%$ \\
\hline
\end{tabular}

Los datos se presentan como mediana (intervalo) o en porcentaje

* Dato calculado en 23 pacientes

Un enfermo recibió esteroides en dosis bajas. Recibieron antimaláricos 17 pacientes.

La mediana del seguimiento de la serie fue de 63 meses (1-316). Al último control, mantuvieron estable la creatininemia de ingreso 21 $(64 \%)$ enfermos, 5 (15\%) la incrementaron en más de $0,30 \mathrm{mg} / \mathrm{dL}$ y 7 (21\%) presentaron creatininemias sostenidamente elevadas entre $3,5 \mathrm{mg} / \mathrm{dL}$ y $5,8 \mathrm{mg} / \mathrm{dL}$. Los pacientes se agruparon según estadios clínicos de función renal, la que se describe para el inicio y el final de la evolución correspondiente (Tabla 2). Hubo dos enfermas con una historia de síntomas de 1 y 2 meses y en quienes se realizó la BR con creatininemia mayor a $3 \mathrm{mg} / \mathrm{d}$. Ambas fallecerían 2 y 3 meses después en hemodiálisis e infección por Acinetobacter.

Al momento de finalizar el estudio, año 2000, había 22 (67\%) enfermos vivos. Once tenían LEG activo con un puntaje en el MEX-SLEDAI mediana: 7 (1-19). Se encontraban en estadio I: 16; en estadio II: 1; en estadio III: 3 y en estadio IV: 2 , de acuerdo con la clasificación en 4 estadios clínicos de función renal en lupus (usa creatini- nemia y proteinuria). Según la clasificación de enfermedad renal crónica K/DOQI, al año 2000 se encontraban en Estadio 1: 7; Estadio 2: 10; Estadio 3: 2; Estadio 4: 2 y Estadio 5: 1; este último paciente en hemodiálisis crónica (Tabla 3). De los 17 pacientes en estadios 1 ó 2, sólo dos tenían proteinuria $>1,5 \mathrm{~g} / \mathrm{d}$.

En 4 pacientes, la BR fue repetida por no presentar síntomas de mejoría. A los 7 años de la primera BR, un enfermo cambió a tipo OMS II, sin embargo fallecería 3 años después de la segunda BR en IRT. En otro caso, a los 3 años no hubo cambio en el tipo $\mathrm{V}$, falleció 9 años después por infección. Otra paciente tuvo 2 biopsias tipo $\mathrm{V}$ puras con un año de diferencia; estaba bien 16 años después. En otro caso, se hizo la segunda biopsia renal 14 años después y cambió a tipo OMS IV; en el año 2000, se encontraba en Estadio 4 de la clasificación K/ DOKI.

Mortalidad. Fallecieron 11 (33\%) enfermos. Los estadios K/DOKI en la fecha cercana al fallecimiento se determinaron en 7 casos: hubo en 
Tabla 2. Estadios clínicos de función renal para enfermos con lupus, al inicio y término del estudio de 33 pacientes con N M L Tipos O M S Va y Vb

\begin{tabular}{|c|c|c|c|c|c|}
\hline \multirow[t]{2}{*}{$\begin{array}{l}\text { Estadios de función renal } \\
\text { al momento de la biopsia renal }\end{array}$} & \multirow[b]{2}{*}{$\mathrm{N}=33$} & \multicolumn{4}{|c|}{$\begin{array}{l}\text { Estadios de función renal } \\
\text { al término del seguimiento }\end{array}$} \\
\hline & & I & II & III & IV \\
\hline $\begin{array}{l}\text { I. Creatininemia }<1,3 \mathrm{mg} / \mathrm{dl} \text { y } \\
\text { proteinuria }<1 \mathrm{~g} / \mathrm{l}\end{array}$ & 2 & 1 & 0 & 1 & 0 \\
\hline $\begin{array}{l}\text { II. Creatininemia }<1,3 \mathrm{mg} / \mathrm{dl} \text { y } \\
\text { proteinuria } \geq 1 \mathrm{~g} / \mathrm{l}\end{array}$ & 26 & 18 & 1 & 4 & 3 \\
\hline $\begin{array}{l}\text { III. Creatininemia entre } 1,3 \text { y } 3 \\
\mathrm{mg} / \mathrm{dL} \text { y cualquier proteinuria }\end{array}$ & 3 & 0 & 1 & 0 & 2 \\
\hline $\begin{array}{l}\text { IV. Creatininemia }>3 \mathrm{mg} / \mathrm{dL} \\
\text { sostenidamente }\end{array}$ & 2 & 0 & 0 & 0 & 2 \\
\hline
\end{tabular}

Clasificación adaptada de Edworthy ${ }^{13,15}$

Tabla 3. Estado de la enfermedad renal crónica al fin del estudio de acuerdo con las $G$ uías «Kidney $D$ isease 0 utcome $Q$ uality Iniatitive (K/D O Q I)» ${ }^{17}$ en 29 pacientes con $N$ M L, según vivo o muerto.

\begin{tabular}{|llccc|}
\hline & Estadios K/DOKI & $\begin{array}{c}\text { Filtración renal } \\
\text { glomerular (FRG) } \\
\text { mL/min* }\end{array}$ & $\begin{array}{c}\text { Pacientes } \\
\text { vivos } \\
(\mathrm{N}=22)\end{array}$ & $\begin{array}{c}\text { Pacientes } \\
\text { fallecidos } \\
(\mathrm{N}=7)^{* *}\end{array}$ \\
\hline Estadio 1 & Daño renal con una FRG normal 0 aumentada & $\geq 90$ & 7 & 2 \\
Estadio 2 & Daño renal con disminución leve de la FRG & $60-89$ & 10 & 0 \\
Estadio 3 & Disminución moderada de la FRG & $30-59$ & 2 & 0 \\
Estadio 4 & Disminución grave de la FRG & $15-29$ & 2 & 2 \\
Estadio 5 & Insuficiencia renal & $<15$ o diálisis & 1 & 3 \\
\hline
\end{tabular}

* Calculado por fórmula de Cockcroft

**No se determinó en 4 fallecidos.

Estadio 1: 2, en Estadio 4: 2 y en Estadio 5: 3 enfermos (Tabla 3). Causas de muerte: complicaciones de la IRT: 3; infección grave: 3; lupus activo: 2 y otras: 3 . Las causas de muerte y la función renal más cercana a la muerte se muestran en la Tabla 4. Una enferma que falleció por causa cardiovascular se había sometido a un trasplante renal a 66 meses de la BR.

Expectativa de vida. Las tasas de supervivencia global estimada fueron de $85 \%$ a 1 año, $75 \%$ a 5 años y 63\% a 10 años. Figura 1.
Análisis univariado. De los factores de riesgo considerados, tuvieron un peor pronóstico los pacientes que al momento de la biopsia renal se encontraban en Estadio clínico renal III versus los que estaban en Estadio II o I, respectivamente (prueba $\log$ rango valor $p=0,000975$ ). Los hipertensos tuvieron también un peor pronóstico (prueba log-rango valor $p=0,03$ ). La creatininemia tuvo un coeficiente del exponente de 1,7; es decir por cada $1 \mathrm{mg}$ de aumento de creatininemia el riesgo aumentó en 70\% (modelo de Cox, valor $\mathrm{p}=$ $0,002)$. 
Rev Méd Chile 2005; 133: 23-32

Tabla 4. Causas de muerte en 11 enfermos con nefropatía membranosa lúpica Tipos O M S Va y Vb.

\begin{tabular}{|c|c|c|c|c|c|c|}
\hline $\begin{array}{l}\text { Causa de } \\
\text { muerte } \\
\text { principal }\end{array}$ & Sexo & $\begin{array}{l}\text { Edad a } \\
\text { la muerte }\end{array}$ & $\begin{array}{l}\text { Duración } \\
\text { del lupus, } \\
\text { meses }\end{array}$ & $\begin{array}{l}\text { Meses de } \\
\text { la BR a la } \\
\text { muerte }\end{array}$ & $\begin{array}{l}\text { LEG activo } \\
\text { a la } \\
\text { muerte }\end{array}$ & $\begin{array}{l}\text { Descripción de las causas de muerte y filtración } \\
\text { renal glomerular (FRG) por Cockcroft o creatininemia. }\end{array}$ \\
\hline \multicolumn{7}{|c|}{ Insuficiencia Renal } \\
\hline & $\mathrm{F}$ & 24 & 5 & 2 & Sí & HD* e infección por Acinetobacter. FRG $9 \mathrm{~mL} / \mathrm{min}$ \\
\hline & $\mathrm{F}$ & 39 & 5 & 3 & Sí & HD e infección por Acinetobacter. FRG $10 \mathrm{~mL} / \mathrm{min}$ \\
\hline & $\mathrm{M}$ & 45 & 186 & 153 & ND料 & $\begin{array}{l}\text { Síndrome edematoso. Insuficiencia renal } \\
\text { (creatininemia 4,1 mg/dLy Clearence } 9 \mathrm{~mL} / \mathrm{min} \text { ) por } 6 \text { meses. Sin HD. }\end{array}$ \\
\hline \multicolumn{7}{|c|}{ Infección Grave } \\
\hline & $\mathrm{F}$ & 29 & 143 & 139 & No & Tuberculosis peritoneal; FRG $97 \mathrm{~mL} / \mathrm{min}$ \\
\hline & $\mathrm{F}$ & 67 & 332 & 260 & No & Meningitis meningocócica; creatininemia $1,3 \mathrm{mg} / \mathrm{dL}$ \\
\hline & $\mathrm{F}$ & 40 & 257 & 94 & No & Septicemia por streptococcus ß hemolítico; creatininemia 0,78 mg/dL \\
\hline \multicolumn{7}{|c|}{ Lupus Eritematoso Generalizado Activo } \\
\hline & $\mathrm{M}$ & 24 & 21 & 1 & Sí & $\begin{array}{l}\text { Lupus pulmonar (derrame pleural) más Aspergilosis pulmonar y } \\
\text { septicemia (aspergillus, staphilococcus epidermidis, enterococcus, } \\
\text { penicilium sp y streptococcus } \alpha \text { viridans). Creatininemia } 1,7 \mathrm{mg} / \mathrm{dL}\end{array}$ \\
\hline & $\mathrm{F}$ & 36 & 65 & 50 & Sí & $\begin{array}{l}\text { Lupus activo renal (hematuria y proteinuria), pulmonar (derrame } \\
\text { pleural) y compromiso cutáneo extenso. FRG } 21 \mathrm{~mL} / \mathrm{min}\end{array}$ \\
\hline \multicolumn{7}{|c|}{ Otras Causas } \\
\hline & M & 60 & 320 & 87 & No & Infarto del miocardio. FRG $99 \mathrm{~mL} / \mathrm{min}$ \\
\hline & $\mathrm{F}$ & 65 & 249 & 240 & No & Infarto del miocardio. Trasplante renal. FRG $26 \mathrm{~mL} / \mathrm{min}$. \\
\hline & $\mathrm{M}$ & 71 & 28 & 6 & No & Cáncer pulmonar. FRG o creatininemia N.D. \\
\hline
\end{tabular}

*HD: hemodiálisis; **ND: no determinado.

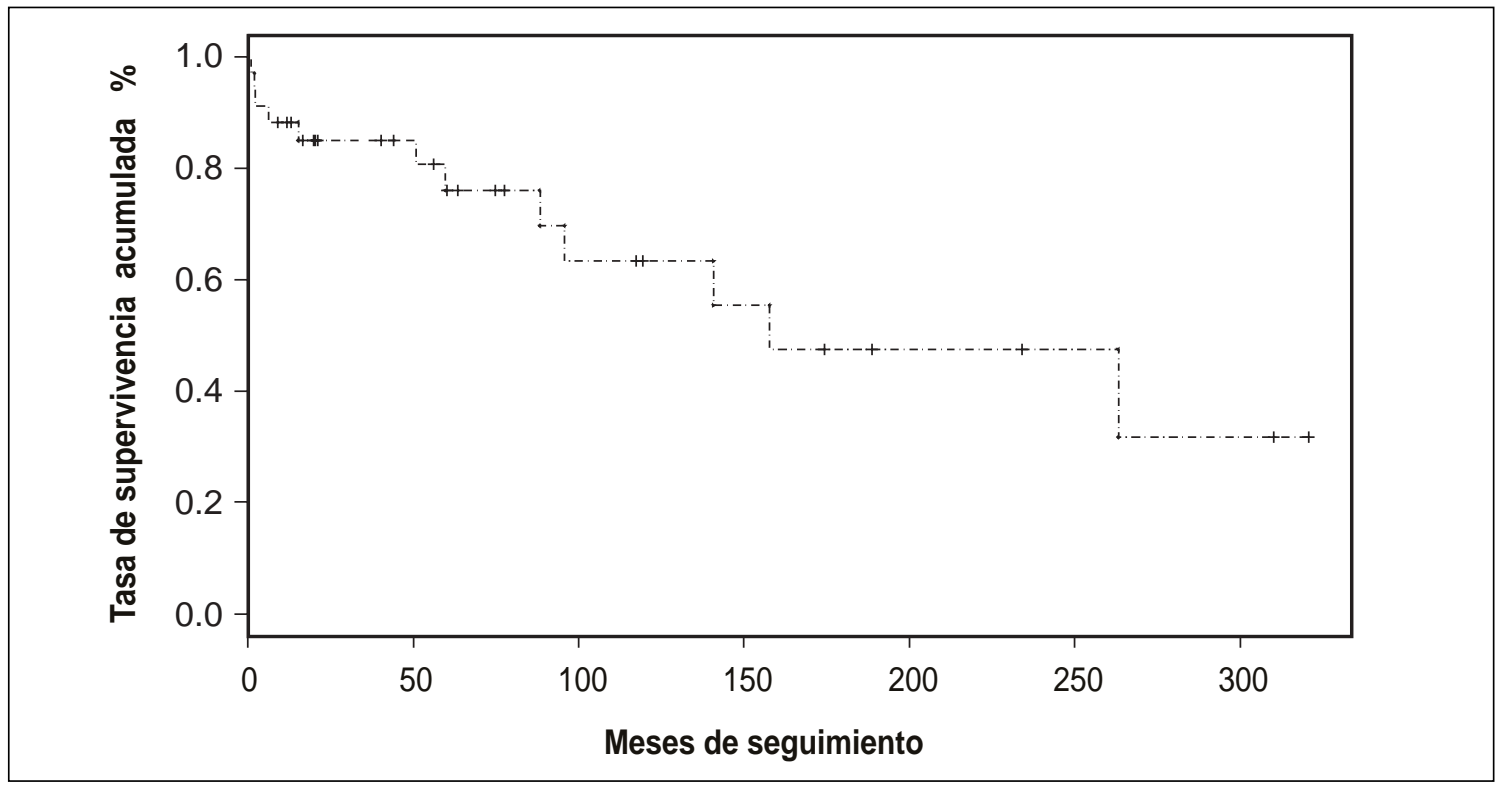

FiguRA 1. Tasa de supervivencia de pacientes con nefritis membranosa lúpica tipos OMS Va y Vb.0 


\section{DisCUSIÓN}

Algunos factores que contribuyen al desacuerdo que existe en la literatura médica sobre la evolución natural y respuesta a terapia de la NML spura». las dificultades de clasificación histológi$\mathrm{ca}^{6,20}$ y un bajo número de enfermos analizados en las series (entre 7 y 36$)^{6,9,10}$, salvo un reciente trabajo que describe 152 pacientes chinos ${ }^{21}$. En un esfuerzo conjunto por dilucidar el pronóstico de estos pacientes, identificamos 33 pacientes de un registro de BR examinadas por un patólogo único, en el curso de 27 años. Si bien este es un estudio retrospectivo del $83 \%$ del total de 40 casos de nefritis $\mathrm{Va} o \mathrm{Vb}$, pensamos que estos resultados contribuyen a un mejor conocimiento de esta patología. La mitad de los 22 enfermos vivos al finalizar el estudio, tenía actividad lúpica al cabo de un seguimiento de mediana 5 años; 17 con buena función renal (depuración de creatinina Cockcroft $\geq 60 \mathrm{~mL} / \mathrm{min}$ y 15 de ellos sin proteinuria relevante); y 5 pacientes con compromiso de la función renal, de los cuales una enferma había cambiado a tipo IV OMS.

Clásicamente, la NML se describe como bien tolerada ${ }^{2}$; así $12 \%$ (8/69) de los pacientes reunidos de 3 series, presentaron IRT después de 5 años de seguimiento promedio $5,6,9,10$. En nuestra serie, $12 \%(4 / 33)$ tenía IRT a 5 años de seguimiento y otro $12 \%$ se encontraba en insuficiencia renal grave (estadio K/DOKI 4), lo que sumado al elevado número de fallecidos (33\%), ilustra el mal curso clínico en nuestro medio. Recientemente una serie española encontró que $42 \%$ de 16 pacientes, tuvo insuficiencia renal crónica a los 8 años de seguimiento promedio ${ }^{8}$. Por otra parte, observamos una tendencia a la mejoría en la supervivencia a 5 años (46\%) entre nuestros primeros diez pacientes con NML publicados en $1994^{13}$ y la serie actual (75\%). Sin embargo, esta última cifra aún es insatisfactoria, si se la compara con la supervivencia estimada en otras series para esta patología (promedia $80 \%$ a 10 años, varía entre $47 \%$ y $90 \%)^{20}$.

El grupo étnico tiene impacto en la supervivencia y respuesta a terapia en lupus, ya que el pronóstico es peor en afro-americanos ${ }^{22}$ y mestizos de América latina ${ }^{23}$ comparados con los caucásicos. Así por ejemplo, la supervivencia a 5 y
10 años de nuestros pacientes con nefritis proliferativa grave ( $\mathrm{N}=26$; 20 tipo IV OMS) tratados con ciclofosfamida IV, fue $82 \%$ y $73 \%$ a 5 y 10 años, respectivamente; con $15 \%$ de IRT ${ }^{24}$. Esto es menor que el estándar de oro del National Institute of Health (NIH) en los Estados Unidos de Norteamérica (EE.UU.), cuya supervivencia es superior a $95 \%$ a 5 años con sólo 3\% de IRT ${ }^{25}$. La expectativa de supervivencia global de nuestros pacientes con NML Va y Vb fue de 75\% a 5 años y 12\% de IRT. Explicaciones posibles para la mala evolución de estos pacientes con nefritis tipo V pura»son: el cambio a nefritis tipo IV, pero esto se demostró sólo en un caso luego de un largo seguimiento; 0 que las terapias indicadas fueron ineficaces, tanto para el síndrome nefrótico como para la NML y la alta tasa de infecciones.

En esta serie, la creatininemia elevada a la biopsia renal y la hipertensión arterial fueron factores de riesgo asociados con un pronóstico peor. La proteinuria en rango nefrótico no lo fue, probablemente porque representaría actividad de la enfermedad y es un factor tratable, en cambio la elevación de la creatininemia reflejaría un daño crónico establecido ${ }^{7}$. Contrario al estudio de Chen y $\mathrm{col}^{21}$, no encontramos diferencias significativas entre pacientes tipo $\mathrm{Va}$ y $\mathrm{Vb}$, probablemente por el número pequeño de enfermos. En ese estudio de 152 pacientes chinos, con NML, examinados entre 1984 y 2001, sólo 20\% se presentó con síndrome nefrótico. Los 91 tipo Vb tuvieron más anemia e hipertensión al inicio y su curso clínico fue peor, 10 duplicaron la creatininemia y 3 tuvieron IRT. En comparación con los 61 tipo Va, donde sólo tres duplicaron la creatininemia y ninguno tuvo IRT ${ }^{21}$.

Hubo muertes tempranas por lupus activo e infección y muertes tardías por IRT, infartos al miocardio e infecciones. La NML en esta serie de pacientes chilenos tuvo una evolución mala con una mortalidad de 33\%, lo que concuerda con otras series $8,11,12$. Las causas de muerte son también similares: infecciones, complicaciones de la insuficiencia renal y cardiovasculares en una proporción menor 7,13,26. En ningún caso se pesquisó trombosis de la vena renal.

No hay acuerdo sobre la terapia «deal» en estos enfermos. A raíz de la gran mejoría obtenida con la terapia con ciclofosfamida IV mensual, en 
pacientes con glomerulonefritis lúpica proliferati$\mathrm{va}^{25}$, ésta se aplicó en NML. Sin embargo, no hay estudios prospectivos de fármacos, doble ciego, con asignación aleatoria y controlados con placebo en pacientes con NML. Algunos autores recomiendan tratamiento precoz de los casos con proteinuria intensa con citotóxicos, con el objeto de reducir las complicaciones del síndrome nefrótico como son la hipercolesterolemia, el edema, la hipercoagulabilidad, la trombosis venosa y la hipertensión ${ }^{5,20}$. Radhakrishnan y col en EE.UU., reportaron la evolución favorable de 7 casos de NML con síndrome nefrótico tratados con dosis bajas de esteroides y ciclosporina $\mathrm{A}^{11}$. Moroni analizó retrospectivamente 19 pacientes con NML y síndrome nefrótico, los 11 enfermos que recibieron mega dosis de Solumedrol ${ }^{\circledR} \mathrm{IV}$, en forma de pulsos combinado con clorambucil, obtuvieron una respuesta más favorable comparado con los 8 pacientes que sólo recibieron corticoesteroides ${ }^{27}$. Chan y col en China, reportaron 20 casos que respondieron en $90 \%$ a dosis altas de esteroides y ciclofosfamida oral, reemplazada luego por azatioprina $^{12}$. Un ensayo todavía muy preliminar, en 41 enfermos, mostró que tanto la ciclofosfamida como la ciclosporina fueron más efectivas en lograr remisión de la proteinuria que los corticoesteroides solos; y se lleva a cabo un ensayo piloto con rapamicina en pacientes con NML pura ${ }^{20}$. Otros autores en cambio, han utilizado esquemas de esteroides en dosis altas en días alternos similar al tratamiento de la nefritis membranosa idiopática ${ }^{20}$.

\section{REFERENCIAS}

1. Churg J, Bernstein J, Giassock RJ. Renal Disease. Classification and Atlas of Glomerular Diseases. Second ed. New York - Tokyo: Igaku-Shoin Medical Publishers, Inc, 1995.

2. Clinical manifestations of systemic lupus erythematosus. In: Ruddy S, Harris E, Sledge C, editors. Kelley's Textbook of Rheumatology. Philadelphia: Saunders, 2001: 1105-23.

3. Golbus J, Mccune WJ. Lupus Nephritis. Classification, prognosis, immunopathogenesis and treatment. Rheum Dis Clin North Am 1994; 20: 213-42.
Nuestros pacientes recibieron dosis altas de esteroides, por al menos un mes, en $81 \%$ de los casos. El tratamiento citotóxico recibido en $58 \%$ de los enfermos fue muy disímil; sólo seis recibieron ciclofosfamida IV y es posible que ésto haya impedido demostrar la utilidad de este citotóxico por sobre los esteroides. Este trabajo no permite recomendar una terapia óptima -como, por ejemplo, agregar citotóxicos a los esteroides en los pacientes con síndrome nefrótico-, por lo que el juicio clínico debe balancear esta modalidad de terapia con sus complicaciones infecciosas, que juegan un papel en la mortalidad observada. Nuestra experiencia es que, en pacientes con lupus, las infecciones son frecuentes, independiente del uso de esteroides o citotóxicos, y ellas se asocian con proteinuria ${ }^{28}$. Por otra parte, el control de la hipertensión arterial puede ser clave para mejorar el pronóstico, como así también tratar el síndrome nefrótico, considerar uso de antagonistas de la angiotensina para minimizar la proteinuria y el uso de estatinas para el tratamiento de las dislipidemias de modo de reducir la magnitud del riesgo cardiovascular a que estos pacientes se encuentran sometidos ${ }^{7,20,29}$. La detección de la glomérulonefritis lúpica, en etapas tempranas, mediante sedimento urinario cada 3 meses y el tratamiento apropiado de la hipertensión son medidas fáciles de llevar a cabo en el cuidado rutinario de los pacientes con $\mathrm{LEG}^{7}$. Mejores recomendaciones para el tratamiento de la NML sólo podrán establecerse en estudios prospectivos multicéntricos con asignación aleatoria de fármacos.

4. Donadio J, Hart G, Bergstralh E, Holley K. Prognostic determinants in lupus nephritis: a long-term clinicopathologic study. Lupus 1995; 4: 109-15.

5. Kolasinski SL, Chung JB, Albert DA. What do we know about lupus membranous nephropathy? An analytic review. Arthritis Rheum 2002; 47(4): 4505.

6. Adier SG, Johnson K, Louie J, Luebling M, Cohen $\mathrm{AH}$. Lupus membranous glomerulonephritis: different prognostic subgroups obscured by imprecise histologic classifications. Modern Pathology 1990; 3(2): 186-91. 
7. ESDAILE JM. How to manage patients with lupus nephritis. Best Pract Res Clin Rheumatol 2002; 16(2): 195-210.

8. Domínguez-Gil B, Hernández E, CarReno A, GonzaÁlez E, Ortiz M, Sierra MP et al. [Diagnostic and clinical course features of lupus membranous glomerulonephritis]. Nefrologia 2000; 20(6): 52331.

9. Pasquali S, Banfi G, Zuccheli A, Moroni G, Ponticew C, Zucche山 P. Lupus membranous nephropathy: long-term outcome. Clin Nephrol 1993; 39(4): 175-82.

10. Sloan R, Schwartz M, Korbet S, Borok R, GROUP AT LNCS. Long-term outcome in systemic lupus erythematosus membranous glomerulonephritis. J Am Soc Nephrol 1996; 7: 229-305.

11. Radhakrishnan J, Kunis Cl, D’agati V, Appel GB. Cyclosporine treatment of lupus membranous nephropathy. Clin Nephrol 1994; 42(3): 147-54.

12. Chan TM, Li FK, Hao WK, Chan KW, Lui SL, Tang $S$ ET AL. Treatment of membranous lupus nephritis with nephrotic syndrome by sequential immunosuppression. Lupus 1999; 8: 545-51.

13. Massardo L, Martinez Me, Jacobem S, Vimarroel L, ROSENBERG H, Rivero S. Survival of Chilean patients with systemic lupus erythematosus. Semin Arthritis Rheum 1994; 24(1): 1-11.

14. The 1982 revised criteria for the classification of systemic lupus erythematosus. Arthritis Rheum 1982; 25: 1271-7.

15. Edworthy S, Bloch D, Mcshane D. A «State Model» for renal function in systemic lupus erythematosus: Its value in the prediction o foutcome in 292 patients. J Rheumatol 1989; 16: 29-35.

16. CоскCROFt DW, Gault MH. Prediction of creatinine clearance from serum creatinine. Nephron 1976; 16(1): 31-41.

17. Levey AS, Coresh J, Balk E, Kausz AT, Levin A, SteFFes MW et al. National Kidney Foundation practice guidelines for chronic kidney disease: evaluation, classification, and stratification. Ann Intern Med 2003; 139(2): 137-47.

18. Guzmán J, Cardiel MH, Arce-Salinas A, SánchezGuerrero J, Alarcón-Segovia D. Measurement of disease activity in systemic lupus erythematosus. Prospective validation of 3 clinical indices. J Rheumatol 1992; 19: 1551-8.

19. Austin HI, Muenz L, Joyce KM, Antonovich T, Kuшck M, Kuppel J et al. Prognostic factors in lupus nephritis: contribution of renal histologic data. Am J Med 1983; 75: 382-91.

20. BaLow JE, Austin HA, 3RD. Therapy of membranous nephropathy in systemic lupus erythematosus. Semin Nephrol 2003; 23(4): 386-91.

21. Chen Q, Lu Z, Hu W, Chen H, Zeng C, Lu L Class V lupus nephritis: a clinicopathologic study in 152 patients. J Nephrol 2003; 16(1): 126-32.

22. Reveide JD, Bartolucci A, Alarcón GS. Prognosis in systemic lupus erythematosus. Negative impact of increasing age at onset, black race, and thrombocytopenia, as well as causes of death. Arthritis Rheum 1990; 33: 37-48.

23. Pons-Estel B, Catoggio L, Cardiel M, Soriano E, VILA A, ABAdi I ET aL. The GLADEL multinational Latin American prospective inception cohort of 1,214 patients with systemic lupus erythematosus. Ethnic and disease heterogeneity among «ispanics». Medicine (Baltimore) 2004; 83: 1-17.

24. Velásquez X, Verdejo U, Massardo L, Martínez M, ARRIAGADA S, ROSENBERG H ET al. Outcome of Chilean patients with lupus nephritis and response to intravenous cyclophosphamide. J Clin Rheumatol 2003; 9: 7-14.

25. SteinBeRg AD, SteinBerg SC. Long-term preservation of renal function in patients with lupus nephritis receiving treatment that includes cyclophosphamide versus those treated with prednisone only. Arthritis Rheum 1991; 34: 945-90.

26. TRAGER J, WARD MM. Mortality and causes of death in systemic lupus erythematosus. Curr Opin Rheumatol 2001; 13(5): 345-51.

27. Moroni G, Maccario M, Banfi G, Quaglini S, Ponticew C. Treatment of membranous lupus nephritis. Am J Kidney Dis 1998; 31(4): 681-6.

28. Massardo L, Martinez ME, Baro M, Figueroa F, Rivero S, Jacobew S. Infecciones en lupus erythematoso generalizado. Rev Méd Chile 1991; 119(10): 1115-22.

29. Cameron J. Lupus nephritis. J Am Soc Nephrol 1999; 10(2): 413-24.

Agradecimientos:

Los autores agradecen a la Sra. Magaly Acuña y Sra. Luzmira Díaz, por su colaboración en reunir las fichas clínicas de los pacientes. 


\begin{abstract}
ADDENDUM
Colaboradores en el estudio de nefritis membranosa lúpica: Drs. Miguel Cuchacovich T. y Héctor Gatica R. Servicio de Reumatología, Hospital Clínico de la Universidad de Chile sosé Joaquín Aguirre», Patricio Downey C. y Roberto Jalil M., Departamento de Nefrología, Hospital Clínico de la Pontificia Universidad Católica de Chile; Juan Carlos Flores H., Servicio de Nefrología del Hospital Militar; Gustavo Fierro, Servicio de Nefrología de Hospital-Clínica INDISA; Enrique Reynolds H., Servicio de Nefrología, Clínica Alemana; Ximena Norambuena R, Servicio de Pediatría, Hospital Ezequiel González Cortés; Elsa Sotomayor, Servicio de Nefrología del Hospital San Juan de Dios; Juan Lemus P., Servicio de Nefrología, Hospital del Salvador, SSO Santiago, Región Metropolitana;
\end{abstract}

Renato Jiménez C., Servicio de Reumatología, Hospital Gustavo Fricke, Viña del Mar; Jorge Vega S., Centro de Diálisis SODINEF y Hospital Gustavo Fricke de Viña del Mar, Quinta Región; Gonzalo Díaz P., Hospital Regional de Talca, Séptima Región; Irene Castro E., Encarnación Sáenz B. y Sergio Recart O., Departamento de Medicina, Universidad de Concepción; Carmen Hernández V., Hospital Regional de Concepción; Juan Carlos Chamorro P., Centro de Diálisis de Los Ángeles; Daniel Enos B., Servicio de Nefrología del Hospital Dr. Víctor Ríos Ruiz, Bío-Bío, Octava Región.

ESTADÍSTICA MSc Paola Viviani G. Centro de Investigaciones Médicas. Escuela de Medicina $\mathrm{dC}: \backslash$ Documents and Settings $\backslash$ Loreto Massardo $\backslash$ Mis documentos $\backslash$ RPtipoVcorre2.doce la Pontificia Universidad Católica de Chile. 